\title{
A validated age and comorbidity prognostic index for survival in dialysis patients
}

\author{
Rodríguez- Mendiola $\mathrm{N}^{1 *}$, Fernández Lucas $\mathrm{M}^{1}$, Teruel $\mathrm{JL}^{1}$, Zamora $\mathrm{J}^{2}$, and Liaño $\mathrm{F}^{1}$ \\ ${ }^{1}$ Department of Nephrology, Ramón y Cajal Hospital, IRyCIS, Madrid, Spain \\ ${ }^{2}$ Department of Biostatistics, Ramón y Cajal Hospital, IRyCIS, Madrid, Spain
}

\section{Introduction}

In 2007, we described a prognostic index (ACPI) for survival in the dialysis population based on 11 comorbid conditions and age [1]. To demonstrate that a predictive model is valuable, evidence that it performs successfully for other groups of patients is needed; this process is called validation [2-6]. We tested the ability of our index to predict the risk of death from comorbid conditions and age, in a cohort comprised of the incident patients acquired by our dialysis centers in the five years following the first study. We here report a validation study, that prospectively evaluated data from a cohort independent of the former test group. We performed this study to assess the reproducibility of the ACPI.

\section{Methods}

\section{Testing population}

The validation was performed using the incident dialysis patients from the same región who started dialysis in the 5 years subsequent to the original study. The cohort consisted of 339 patients with endstage renal disease (ESRD) who began chronic haemodialysis between January 2003 and December 2008. The period of observation was through December 2010, in order to guarantee a theoretical follow-up of at least 2 years. Patients were censored at the time of transplantation or study end. Actuarial survival analysis included those who died in the first 90 days.

Patient comorbidity and epidemiology data at the start of dialysis were obtained prospectively from the electronic records of our dialysis centers, following a predefined protocol by the same group of physicians who participated in the original study.

\section{Statistical analysis}

For statistical analysis, SPSS 15.0 was used. We calculated the frequencies of comorbid conditions in the testing population. A Cox proportional hazards mode 1 was fitted to construct univariate and multivariate analysis [7].

Each patient was scored with the sum of ACPI's weighted conditions (Table I) and classified into three levels of mortality risk (low, medium, and high). Conditions were We calculated each group's probability of survival according to the Kaplan-Meier method and compared their results by log-rank test [7]. Analysis with receiver operating characteristic (ROC) curve [7] was performed to assess the discrimination of our index on the tested sample.

\section{Results}

\section{Testing cohort characteristics}

The study population included 339 patients with ESRD who had started haemodialysis. There were 113 female patients (33.3\%). The average age of the total cohort was with $63 \pm 14$ years (range 20-86) with $41 \%$ aged 70 years or older. The median follow-up was $27 \pm 21$ months (range 1-85). The most frequent cause of nephropathy were interstitial (21.2\%) and DM (20.9\%). Forty-two patients had chronic renal allograft dysfunction.

The frequency and hazard ratio (HR) of each comorbidity in the univariate analysis and the crude mortality rate are shown in Table II. The prevalence of comorbid conditions ranged from $37.8 \%$ for noncontrolled hypertension to $4.4 \%$ for systemic diseases. Both ischemic heart disease with symptoms of chronic heart failure and symptoms of chronic heart failure (CHF) due to other cardiopathy, scored the highest risk of death.

We calculated the adjusted relative risk of death with each comorbid condition that had been established by the ACPI index. The HRs found in the multivariate model are shown in Table III. Ischemic heart disease with CHF triples the risk of death in an independent manner, and CHF without ischemic heart disease double the risk. In contrast, ischemic heart disease without CHF is not associated with increased risk of mortality. Peripheral vascular disease doubles the risk of mortality and systemic diseases triple it. The remaining conditions obtained a HR with a confidence interval that included hypothesis null $(\mathrm{HR}=1)$, thus they were not statistically significant.

\section{Testing cohort mortality}

The global mortality rate was found to be $36.9 \%$ (125/339); the causes of death are shown in Table IV. The leading causes of death were cardiac and vascular events (peripheral and cerebral), accounting for $24 \%$ of all deaths. Infectious processes were responsible for $16 \%$ of deaths.

A significant difference in mortality rate was not found between patients with previous kidney transplant (38.1\% [16/42]) and transplant naive patients $(36.7 \%[109 / 297]), \mathrm{p}=0.47$.

Correspondence to: Nuria Rodríguez Mendiola. Hospital Ramón y Cajal, Ctra Colmenar Viejo Km.9.100, Madrid 28037, Spain, Tel: 913368018, 639290497; E-mail: nurirodrimendiola@yahoo.es.

Received: February 10, 2017; Accepted: March 02, 2017; Published: March 06 2017 
Table 1. Assigned weights for age and diseases in the ACPI scoring.

\begin{tabular}{|l|c|}
\hline Conditions & ACPI weight \\
\hline Age $<50$ years & 0 \\
\hline $50-59$ years & 2 \\
\hline $60-69$ years & 3 \\
\hline$>70$ years & 3 \\
\hline Ischemic heart disease without chronic heart failure & 0 \\
\hline Ischemic heart disease with chronic heart failure & 3 \\
\hline Noncontrolled arterial hypertension & 1 \\
\hline Atrial fibrillation & 1 \\
\hline Chronicobstructivepulmonarydisease & 1 \\
\hline Gastrointestinal disease & 1 \\
\hline Systemicdisease & 2 \\
\hline Diabetes mellitus & 2 \\
\hline Chronichepatopathy & 2 \\
\hline Congestiveheartfailure & 2 \\
\hline Peripheral vascular disease & 2 \\
\hline Malignancy of $<5$ years of evolution & 4 \\
\hline
\end{tabular}

Table 2. Prevalence, univariate analysis and crude mortality rates of each disease.

\begin{tabular}{|c|c|c|c|}
\hline Comorbidcondition & Number (\%) & HR (95\% CI) & Mortalityrate, n (\%) \\
\hline $\begin{array}{c}\text { Cardiopathy: } \\
\text { Ischemic heart disease }\end{array}$ & $89(26.2)$ & & \\
without chronic heart failure & $33(9.7)$ & $1.10(0.63-1.92)$ & $15(45.5)$ \\
$-\quad \begin{array}{c}\text { Ischemic heart disease } \\
\text { with chronic heart failure }\end{array}$ & $22(6.5)$ & $4.21(2.34-7.54)$ & $14(63.6)$ \\
$-\quad \begin{array}{c}\text { Chronic heart failure } \\
\text { without ischemic heart disese }\end{array}$ & $34(10.0)$ & $2.24(1.38-3.64)$ & $21(61.8)$ \\
\hline Peripheral vascular disease & $62(18.3)$ & $1.93(1.32-2.82)$ & $39(62.9)$ \\
\hline Cerebral vascular disease & $47(13.9)$ & $1.29(0.83-2.02)$ & $24(51.1)$ \\
\hline Diabetes mellitus & $108(31.9)$ & $1.20(0.84-1.73)$ & $46(42.6)$ \\
\hline Noncontrolledhypertension & $128(37.8)$ & $0.99(0.69-1.42)$ & $50(39.1)$ \\
\hline Atrial fibrillation & $43(12.7)$ & $1.70(1.09-2.67)$ & $24(55.8)$ \\
\hline Chronicpulmonarydisease & $49(14.5)$ & $1.99(1.29-3.04)$ & $27(55.1)$ \\
\hline Liverdisease & $38(11.2)$ & $1.31(0.77-2.2)$ & $16(42.1)$ \\
\hline Gastrointestinal disease & $61(18.0)$ & $0.93(0.60-1.44)$ & $26(42.6)$ \\
\hline Systemicdisease & $15(4.4)$ & $2.47(1.29-4.71)$ & $10(66.7)$ \\
\hline Malignancy<years & $35(10.3)$ & $1.47(0.88-2.46)$ & $17(48.6)$ \\
\hline
\end{tabular}

\begin{tabular}{|c|c|}
\hline Comorbidcondition & HR (95\% IC) \\
\hline $\begin{array}{c}\text { Cardiopathy: } \\
\text { - Ischemic heart disease without chronic heart failure } \\
\text { - Ischemic heart disease with chronic heart failure }\end{array}$ & $0.99(0.55-1.77)$ \\
- Chronic heart failure without ischemic heart disease & $3.67(1.88-7.19)$ \\
\hline Peripheral vascular disease & $2.21(1.30-3.77)$ \\
\hline Noncontrolledhypertension & $1.80(1.16-2.79)$ \\
\hline Hypertension & $1.04(1.04-3.0)$ \\
\hline Chronic atrial fibrillation & $1.0(0.68-1.46)$ \\
\hline Chronicpulmonarydisease & $1.04(0.62-1.74)$ \\
\hline Chronicliverdisease & $1.37(0.85-2.21)$ \\
\hline Gastrointestinal disease & $1.65(0.94-2.87)$ \\
\hline Systemicdisease & $0.87(0.55-1.38)$ \\
\hline Malignancy $<5$ years & $3.22(1.62-6.41)$ \\
\hline
\end{tabular}

Table 4. Causes of death

\begin{tabular}{|c|c|c|}
\hline Cause of death & Number of cases & Proportion (\%) \\
\hline Cardiacdisease & 30 & 24 \\
\hline Vascular disease & 30 & 24 \\
\hline Infectious disease & 20 & 16 \\
\hline Malignant neoplasm & 16 & 12.8 \\
\hline
\end{tabular}

\section{Application of ACPI on testing population}

We applied the ACPI scoring system to the study cohort and found the mean global score was $5.26 \pm 3$ (range $0-15$ ). There was no difference between those who had undergone a previous kidney transplant (score 4.98 [range 0-12]) and those who had not (score 5.32 [range 0-15]), with a significance of $P=0.83$. The distribution of patients among risk groups was: $35(10.3 \%)$ patients with low risk (0-1 points); 115 patients (33.9\%) with medium risk, and 189 patients (55.8\%) with high risk.

The 5-year survival rates of the three risk levels are shown in Figure 1. The global mortality rate per 100 patients per year was $16 / 100$. We also calculated it in the three risk strata defined by our index, the results of which were 10.4/100 patients per year in the medium risk group and 23.3/100 patients per year in the high risk group. There were no deaths in the low risk group.

\section{Comparison with the Charlson Index}

To compare the predictive power of our survival model with the Charlson index [8], we used C statistics, Harrell's C discrimination index [9], and the Cox proportional hazards model.

The $\mathrm{C}$ statistics for the relation between our index and the Charlson index [8] were similar for the logistic models predicting a 5-year mortality rate on our validation cohort (Figure 2). The ROC curves showed the following areas under the curve: IPEC 0.733 (IC 95\%, 0.680-0.787) and Charlson 0.751 (IC 95\%, 0.700-0.803) (Figure II). We calculated Harrell's C index and obtained risk estimates, stratified by risk levels, by Cox proportional hazard regression model for our index and the Charlson index, as shown in Table 5 Harrell's C index [9] was very similar to both ACPI and Charlson, and with a value close to 0.7 , which supports a model's predictive adequacy. Both indexes revealed that the relative risk of death for each increasing level of the comorbidity index was $19 \%$ in the ACPI index and $30 \%$ in the Charlson index.

\section{Discussion}

The Charlson comorbidity [8] score is widely used to assess the outcome of patients on dialysis therapy, although it was neither designed nor validated for a population with ESRD. This index was designed on patients admitted in a medical institution with different diagnoses and validated in a cohort of breast cancer patients, populations which are not comparable with those patients with ESRD. Hemmerlgan et al. [10] adapted the Charlson index in an incident dialysis population, but the influence of age on mortality was not evaluated. Our index solves these limitations by combining both comorbid conditions and age in an ESRD population.

The main limitation of our index was the lack of validation. To assess the reproducibility of a prognostic index, it needs to be validated in a different cohort of patients from the original study [2-6 ]. It is important to create a validated age and comorbidity index for ESRD patients in order to predict survival and for risk adjustment in dialysis cohort studies. Therefore, we tested the ability of our index to predict risk of death from comorbid diseases and age in a cohort of 5-year subsequent incident patient population.

The general characteristics of our present study population were very similar to the original cohort. Up to $50 \%$ of individuals in both cohorts scored five points or higher. This fact solves one of the Charlson index [8] limitations; it was defined on a population admitted to medical services with a lower burden of comorbidity than an incident dialysis population.

Our findings about comorbidity risk weigh on testing cohort substantiates which was suggested by our ACPI index. Heart disease scores highly in our index, and is the development of heart failure symptoms what have a relevant role in ESRD patients for long-term 


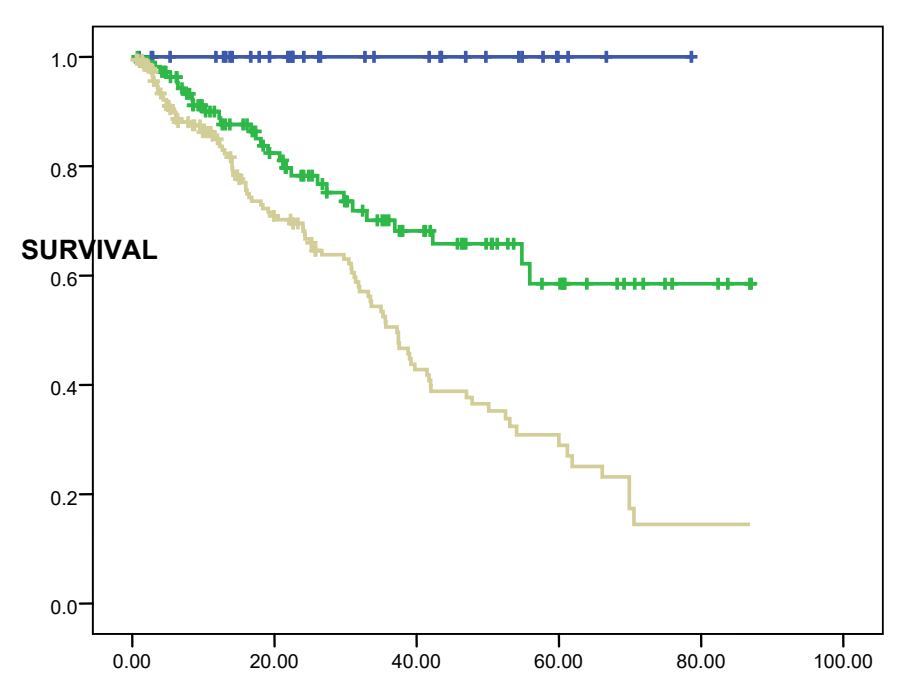

Figure1. Kaplan-Meier curves of the 3 risk levels defined by the age-comorbidity prognostic index (ACPI). Log-rank test: 33,$5 ; \mathrm{p}=0.000$.

\section{ROC CURVE}

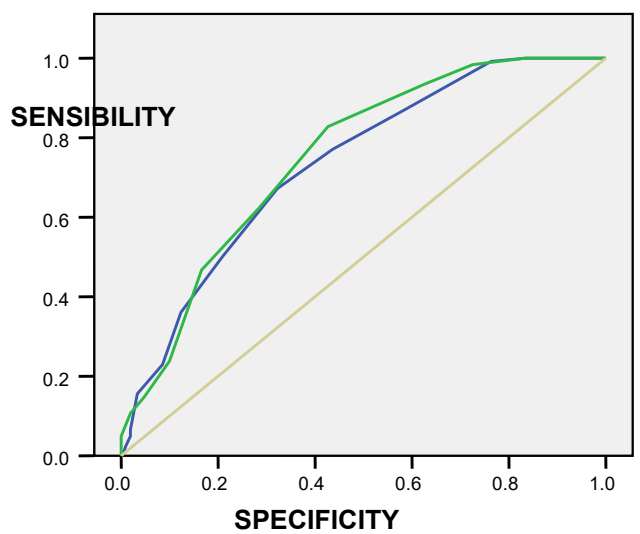

Figure 2. Comparison of receiver operating characteristics (ROC) curves for predicting mortality, among ACPI and Charlson. Areas under the curve: ACPI 0.733 (IC 95\% 0.6800.787 ) andCharlson 0.751 (IC 95\% 0.700-0.803).

survival $[1,12]$. Atrial fibrillation, not often included in most used comorbid indexes $[8,10,13,14]$ is a relevant condition that increases mortality risk in both cohorts. Both peripheral vascular disease and chronic obstructive pulmonary disease also showed a negative impact on survival. Vascular peripheral disease obtains a higher risk in our index than in others $[8,10,13]$ by looking at our current practice, we noticed that when vascular disease turns into several clinical complications, it has a negative impact on our patients' survival. Moreover, our index points out the high mortality of patients with previous neoplasm, but only in those cases with less than 5 years' evolution since diagnosis. Only one relevant difference was found regarding uncontrolled hypertension, which showed less impact on the study population's mortality.

Cause of death and global 5-year mortality rate were similar in both cohorts. There was no difference between high and medium strata mortality rates either, but no individual died in the present cohort's low risk group. Therefore, this supports the adequate stratification of patients into risk groups when we apply the ACPI on the present study's population.
Our index showed a satisfactory predictive power when we evaluated it on the present study's population. The ROC curves and Harrell's $C$ index were very similar when calculated in the original and the testing cohorts. We also determined both parameters for the Charlson index on both populations and their results were also very similar, and very close to those obtained with our index. This fact supports the ability of both indexes.

There are notable features that make our ACPI index valuable. It has been elaborated and validated on a specific incident dialysis population with a high burden of comorbidity and takes age into account. A unique group of physicians collected our patients' epidemiology and comorbidity data at the start of dialysis in a prospective manner, contrary to those indexes elaborated from administrative data [15-17]. Our index is a simple one that analyses just 11 conditions and four age groups at the start of dialysis. It takes into account conditions that other indexes do not. The ACPI scoring system is easily applicable and allows patient stratification into three risk groups with a 5-year estimated mortality, a period of time that fits with the medium survival of haemodialysis incident patients. This has been validated in a different patient cohort than that used in the original study, in a temporal validation $[3,4,18]$ that provides an adequate grade of reproducibility.

There is, however, a limitation to our study. Our validation cohort was comprised of incident patients from the same center in which our original study was performed and who began dialysis in the 5 -year period following the completion of the first study. Therefore, the current study provides an external validation in a temporal sense, but it could be considered an intermediate [19], between internal and external validation, because the individuals were collected from the same centre. This is notable, especially from a statistical perspective, but it is not as relevant from a clinical point of view.

\section{References}

1. Fernandez Lucas M, Teruel JL, Zamora J, Lopez Mateos M, Rivera M, et al. (2007) A Mediterranean age-comorbidity prognostic index for survival in dialysis populations. $J$ Nephrol 20: 696-702. [Crossref]

2. Moons KG, Altman DG, Vergouwe Y, Royston P (2009) Prognosis and prognostic research: application and impact of prognostic models in clinical practice. BMJ 338: b606. [Crossref]

3. Altman DG, Royston P (2000) What do we mean by validating a prognostic model? Stat Med 19: 453-473. [Crossref]

4. Altman DG, Vergouwe Y, Royston P, Moons KG (2009) Prognosis and prognostic research: validating a prognostic model. BMJ 338: b605. [Crossref]

5. Bleeker SE, Moll HA, Steyerberg EW, Donders AR, Derksen-Lubsen G, et al. (2003) External validation is necessary in prediction research: a clinical example. $J$ Clin Epidemiol 56: 826-832. [Crossref]

6. Royston P, Parmar MK, Sylvester R (2004) Construction and validation of a prognostic model across several studies, with an application in superficial bladder cancer. Stat Med 23: 907-926. [Crossref]

7. Martínez-González MA, Sánchez-Villegas A, Faulin Fajardo J (2008) Bioestadística amigable. ( $3^{\mathrm{a}}$ ed), Navarra: Martinez-González MA.

8. Charlson ME, Pompei P, Ales KL, MacKenzie CR (1987) A new method of classifying prognostic comorbidity in longitudinal studies: development and validation. $J$ Chronic Dis 40: 373-383. [Crossref]

9. Harrell FE Jr, Lee KL, Mark DB (1996) Multivariable prognostic models: issues in developing models, evaluating assumptions and adequacy, and measuring and reducing errors. Stat Med 15: 361-387. [Crossref]

10. Hemmelgarn BR, Manns BJ, Quan H, Ghali WA (2003) Adapting the Charlson Comorbidity Index for use in patients with ESRD. Am J Kidney Dis 42: 125-132. [Crossref]

11. Lucas MF, Quereda C, Teruel JL, Orte L, Marcén R, et al. (2003) Effect of hypertension before beginning dialysis on survival of hemodialysis patients. Am J Kidney Dis 41: 814-821. [Crossref] 
12. Wang AY, Sanderson JE (2011) Current perspectives on diagnosis of heart failure in long-term dialysis patients. Am J Kidney Dis 57: 308-319. [Crossref]

13. Khan IH, Catto GR, Edward N, Fleming LW, Henderson IS, et al. (1993) Influence of coexisting disease on survival on renal-replacement therapy. Lancet 341: 415-418. [Crossref]

14. Davies SJ, Phillips L, Naish PF, Russell GI (2002) Quantifying comorbidity in peritoneal dialysis patients and its relationship to other predictors of survival. Nephrol Dial Transplant 17: 1085-1092. [Crossref]

15. Liu J, Huang Z, Gilbertson DT, Foley RN, Collins AJ (2010) An improved comorbidity index for outcome analyses among dialysis patients. Kidney Int 77: 141-151. [Crossref]
16. Couchoud C, Labeeuw M, Moranne O, Allot V, Esnault V, et al. (2009) A clinical score to predict 6-month prognosis in elderly patients starting dialysis for end-stage rena disease. Nephrol Dial Transplant 24: 1553-1561. [Crossref]

17. Miskulin D, Bragg-Gresham J, Gillespie BW, Tentori F, Pisoni RL, et al. (2009) Key comorbid conditions that are predictive of survival among hemodialysis patients. Clin $J$ Am Soc Nephrol 4: 1818-1826. [Crossref]

18. Steyerberg EW, Bleeker SE, Moll HA, Grobbee DE, Moons KG (2003) Internal and external validation of predictive models: a simulation study of bias and precision in small samples. J Clin Epidemiol 56: 441-447. [Crossref]

19. Khan IH, Catto GR, Edward N, Fleming LW, Henderson IS, et al. (1993) Influence of coexisting disease on survival on renal-replacement therapy. Lancet 341: 415-418. [Crossref]

Copyright: (C2017 Mendiola NR. This is an open-access article distributed under the terms of the Creative Commons Attribution License, which permits unrestricted use, distribution, and reproduction in any medium, provided the original author and source are credited. 